Mutation Research/Genetic Toxicology and Environmental Mutagenesis

Volume 744, Issue 2, 15 May 2012, Pages 161-166

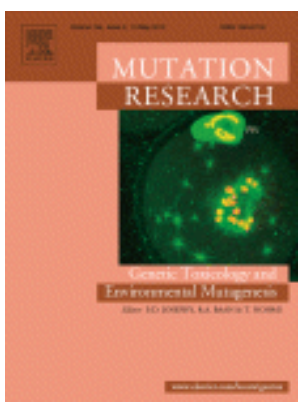

\title{
Re-evaluation of a reported increased micronucleus frequency in lymphocytes of workers occupationally exposed to formaldehyde
}

- Günter Speit ${ }^{\mathrm{a}, \mathbf{A}}, \mathbf{\nabla}$,

- Carina Ladeira ${ }^{\mathrm{b}}$,

- Regina Linsenmeyer ${ }^{a}$,

- Petra Schütz

- $\underline{\text { Josef Högel }}{ }^{\mathrm{a}}$

- a Institut für Humangenetik, Universität UIm, D - 89069 Ulm, Germany

- ${ }^{b}$ Escola Superior de Tecnologia da Saúde de Lisboa, Instituto Politécnico de Lisboa, Lisbon, Portugal

\section{Abstract}

A replicate evaluation of increased micronucleus (MN) frequencies in peripheral lymphocytes of workers occupationally exposed to formaldehyde (FA) was undertaken to verify the observed effect and to determine scoring variability. May-GrünwaldGiemsa-stained slides were obtained from a previously performed cytokinesis-block micronucleus test (CBMNT) with 56 workers in anatomy and pathology laboratories and 85 controls. The first evaluation by one scorer (scorer 1) had led to a highly significant difference between workers and controls (3.96 vs $0.81 \mathrm{MN}$ per 1000 cells). 
The slides were coded before re-evaluation and the code was broken after the complete re-evaluation of the study. A total of 1000 binucleated cells (BNC) were analysed per subject and the frequency of MN (in \%o) was determined. Slides were distributed equally and randomly between two scorers, so that the scorers had no knowledge of the exposure status. Scorer 2 (32 exposed, 36 controls) measured increased $\mathrm{MN}$ frequencies in exposed workers (9.88 vs 6.81). Statistical analysis with the two-sample Wilcoxon test indicated that this difference was not significant ( $p=0.17$ ). Scorer 3 (20 exposed, 46 controls) obtained a similar result, but slightly higher values for the comparison of exposed and controls (19.0 vs $12.89 ; p=0.089$ ). Combining the results of the two scorers (13.38 vs 10.22), a significant difference between exposed and controls $(p=0.028)$ was obtained when the stratified Wilcoxon test with the scorers as strata was applied. Interestingly, the re-evaluation of the slides led to clearly higher $\mathrm{MN}$ frequencies for exposed and controls compared with the first evaluation. Bland-Altman plots indicated that the agreement between the measurements of the different scorers was very poor, as shown by mean differences of 5.9 between scorer 1 and scorer 2 and 13.0 between scorer 1 and scorer 3 . Calculation of the intra-class correlation coefficient (ICC) revealed that all scorer comparisons in this study were far from acceptable for the reliability of this assay. Possible implications for the use of the CBMNT in human biomonitoring studies are discussed.

\section{Keywords}

- CBMNT;

- May-Grünwald-Giemsa-staining;

- Coefficient of variation;

- Intra-class correlation coefficient 